\title{
Questes
}

vestes Revue pluridisciplinaire d'études médiévales

$10 \mid 2006$

Croire

\section{Compte rendu du colloque « Sommeil, songes et insomnies »}

Sophie Albert

\section{(2) OpenEdition}

1 Journals

Édition électronique

URL : http://journals.openedition.org/questes/327

DOI : $10.4000 /$ questes.327

ISSN : 2109-9472

Éditeur

Les Amis de Questes

Édition imprimée

Date de publication : 15 novembre 2006

Pagination : 48-54

ISSN : 2102-7188

\section{Référence électronique}

Sophie Albert, "Compte rendu du colloque « Sommeil, songes et insomnies » », Questes [En ligne], 10 | 2006, mis en ligne le 01 janvier 2014, consulté le 25 septembre 2020. URL : http:// journals.openedition.org/questes/327 ; DOI : https://doi.org/10.4000/questes.327

(C) Association des amis de «Questes » 


\section{Sommeil, songes et insomnies}

\section{Colloque de la Société de Langue et de Littérature Médiévales d'Oc et d'Öil}

\section{Université de Rennes II, 28-29 septembre 2006 compte rendu rédigé par Sophie ALBERT}

L’Université de Rennes II hébergeait cette année le colloque bisannuel de la Société de Langue et de Littérature Médiévales d'Oc et d'Oïl, organisé conjointement par Elisabeth Gaucher, Christine Ferlampin-Acher et Denis Hüe. Sans prétendre rendre compte en détail de chaque communication, je vous propose ici, en manière de "mise en bouche», un résumé ordonné de la rencontre.

Bien que l'intitulé du colloque, «Sommeil, songes et insomnies », décline trois facettes de la vie nocturne et des relations entre veille et sommeil, la plupart des communications se concentrent sur l'un ou l'autre aspect. Quatre traitent de divers gestes ou phénomènes physiologiques associés à l'ensommeillement.

Jean-Marie Fritz examine, à travers plusieurs textes littéraires, les sons accompagnant l'entrée dans le sommeil. Il souligne la récurrence des mentions du chant des oiseaux et du murmure de l'eau, relevant tous deux de la topique $\mathrm{du}$ locus amoenus. Après avoir passé en revue quelques constantes des descriptions de bruits artificiels, musique instrumentale ou mélopée maléfique de la sirène, il confronte les textes littéraires à plusieurs « régimes de santé » médiévaux. Si les berceuses y sont fort rares, fréquente en revanche est la présence d'une eau somnifère; elle se justifie par un arrière-plan théorique d'obédience aristotélicienne, qui voit dans le sommeil une combinaison du froid et de l'humide. Liée à la mort aussi bien qu'au sommeil, l'eau somnifère 
constitue ainsi un point de convergence intéressant entre les textes médicaux et la littérature. A côté ou en marge de ces théories médicales, l'Ovide moralisé, étudié par Marylène Possamaï-Pérez, fait une large place, dans les procédés conduisant au sommeil, à la ruse et à la magie. Le traducteur français présente Mercure, plutôt que Morphée, comme un grand manipulateur de sommeil, qui endort ses victimes par sa musique (Livre I) et, surtout, par sa crosse magique (Livre II). Aussi le sommeil, dans la moralisation médiévale, demeure-t-il fondamentalement ambivalent, tout comme la métamorphose à laquelle l'ensommeillement emprunte ses modes d'expression.

Les deux autres communications, quoique fort différentes, avaient pour point commun de rechercher, dans des textes médiévaux, la trace de rituels antiques. Jean-Jacques Vincensini, à travers un «périple lexico-culturel» autour du verbe « bâiller », montre que la définition purement physiologique du mot s'avère insuffisante; sous-tendu par des souvenirs de rites païens pour lesquels le bâillement était une porte ouverte à l'échappée de l'âme, le sens du geste comprend une dimension existentielle à laquelle les traducteurs doivent rester attentifs. Le lien paraît indissociable entre le sommeil et la mort, le souffle et l'esprit. De son côté, partant du rituel antique du songe d'incubation dont le Roman de Brut fournit un exemple isolé, mais significatif (v. 675-678), Fabienne Pomel tente d'en déceler la trace dans la littérature médiévale. Le modèle, fortement laïcisé, donne lieu à deux types de scénarios : un scénario courtois, dans lequel le songe est occasionné par une méditation ou une littérale incubation sur un objet de l'amour; un scénario studieux, où le songe, dans l'austérité de la bibliothèque, est provoqué par une lecture édifiante. Quant à la fonction thaumaturge $\mathrm{du}$ rêve d'incubation, elle trouve un écho dans l'apparition, dans le cadre du songe, d'une personne salvatrice ou consolatrice. Fabienne Pomel note pour finir que le songe figure souvent le lieu d'incubation de l'œuvre, le rituel préliminaire ayant pour objet de désigner les sources à partir desquelles le texte se construit. 
A l'opposé, en quelque sorte, de ces procédés entraînant le sujet vers un sommeil profond, l'insomnie, inversion du rapport naturel entre la nuit et le jour, fait l'objet de deux interventions. Comme l'explique Nathalie Koble, la nuit est dans le Livre d'Artus le moment de batailles adoptant la topique descriptive de la chanson de geste et, surtout, le moment des émois érotiques d'Arthur autant que de Gauvain et d'autres chevaliers. Dans la multiplication de ces insomnies amoureuses, Nathalie Koble lit les premières étapes d'un parcours initiatique, encore inachevé dans cette suite rétrospective au cycle du Lancelot-Graal, menant de l'emprise des sens à la maîtrise courtoise. Sébastien Douchet, pour sa part, étudie l'insomnie d'Arthur dans l'économie narrative de la sixième branche de la Première Continuation: appel à la merveille lancé au roi par l'Autre Monde, l'insomnie transforme Arthur, détenteur d'un savoir ignoré de son entourage, en maître de cérémonie du récit à venir ; elle entretient un rapport de spécularité avec la branche précédente, dans laquelle Gauvain péchait à force de sommeil. Cette spécularité entre à son tour en résonance avec le mode de fonctionnement strictement symétrique de la vengeance de Guerrehet, dont la narration occupe la majeure partie de la sixième branche.

S'attachant à un phénomène situé à la croisée du sommeil et de la veille, huit communications sont consacrées à l'univers onirique. Si l'ensemble de ces interventions vise à exposer les modalités d'apparition et de fonctionnement du songe et, par ce biais, à éclairer différentes logiques textuelles, toutes ne donnent pas la même extension à leur domaine d'application. Alain Corbellari, réfléchissant sur le rapport entre «récits de rêves et genres littéraires dans la littérature française du XIII ${ }^{\mathrm{e}}$ siècle», pose les jalons d'une réflexion générique sur le songe. Très stéréotypé dans les premières chansons de geste, le songe devient plus souple et inventif dans les chansons empruntant au roman ou au conte merveilleux ; frappé de discrédit dans les romans en vers du XII ${ }^{\mathrm{e}}$ siècle, il 
revient timidement chez les épigones de Chrétien de Troyes ; enfin, tourné en dérision dans le fabliau et le corpus renardien, il y retranscrit une vision outrancièrement matérialiste du réel et une idéologie foncièrement misogyne. Dans une perspective tout aussi large mais qui, à bien des égards, s'oppose à toute classification générique, Jelles Koopmans tente de cerner les contours, nécessairement fuyants, d'un univers onirique dépassant les barrières des seuls récits de rêve. Il voit dans toutes les formes de transgression, notamment alimentaires, que déploie la littérature du $\mathrm{XV}^{\mathrm{e}}$ siècle, la présence d'un univers onirique lié à des formes plus ou moins ritualisées d'inversion des valeurs.

Plusieurs communications peuvent être considérées comme des « études de cas » susceptibles d'illustrer l'un ou l'autre de ces vastes aperçus. Quoique daté du début du XIII ${ }^{\mathrm{e}}$ siècle, le Songe d'Enfer de Raoul de Houdenc, dont Chantal Connochie-Bourgne propose une analyse, s'inscrit dans un imaginaire de l'abondance proscrite qui n'est pas sans faire écho aux remarques de Jelles Koopmans; que cet imaginaire coexiste avec une manifeste intention didactique, visible par exemple dans l'échelle de vices que parcourt le rêveur, souligne la singularité de ce récit de songe. De leur côté, Bernard Guidot pour le cycle de Guillaume d'Orange, Armand Strubel pour le songe de Chanteclerc dans la Branche II du Roman de Renart, étayent les perspectives ouvertes par Alain Corbellari, en précisant les modes de fonctionnement du songe dans un contexte générique donné. Les songes du cycle de Guillaume, ponctuant généralement un moment stratégique du récit, témoignent d'un rapport immédiat au divin; leur langage, sans pour autant dégager systématiquement des leçons univoques, emprunte à la symbolique la plus conventionnelle. Quant au songe de Chanteclerc, il introduit un écart stylistique maximal entre le lieu de la narration, une prosaïque basse-cour, et la virtuose technique exégétique que convoque la poule Pinte. Le rêve, en un parcours inédit, réussit à dire l'évidence en la dissimulant juste assez pour que Chanteclerc s'entête à ne pas la 
comprendre. Tout en exploitant les formes habituelles du comique renardien, le passage pervertit le rapport entre rêve et réalité.

Les communications de Mireilles Demaules et de Gérard Gros concernent un domaine évoqué seulement par Alain Corbellari, le roman arthurien en prose. Gérard Gros, s'attachant au songe de Mordrain dans l'Estoire del saint Graal, en dégage les enjeux dynastiques et idéologiques ; il montre comment se définit la place, dans l'ordre de la chair et de l'esprit, de chaque membre de la lignée. Mireille Demaules, pour sa part, établit que le Lancelot en prose assure aux rêves amoureux une nette promotion, les créditant d'une vérité aussi bien prophétique que psychologique ; l'examen de leur structure et de leur fonction au sein du récit-cadre conduit cependant à distinguer les rêves de Guenièvre et de Lancelot, unis tout à la fois par leur similitude et par le type d'amour qui s'y trouve exprimé, du rêve de Galehaut, représentant un amour héroïque hérité de genres plus anciens - chanson de geste et roman d'antiquité - et appelé à disparaitre $\mathrm{du}$ monde romanesque et courtois. Cette distinction trouve son corollaire dans la présence de deux modes d'écriture : au rêve de Galehaut, qui use d'un bestiaire allégorique tributaire de la chanson de geste, s'oppose la transparence des rêves de Guenièvre et de Lancelot, signe d'une modernité liée à la nouvelle écriture romanesque.

Deux communications enfin, celles de Paul Bretel et de Jean-René Valette, déterminent la place des songes au sein d'une hiérarchie plus vaste d'attitudes vis-à-vis du sommeil. Paul Bretel insiste sur la suspicion qui, dans les contes de la Vie des Pères et le Gracial d'Adgar, pèse sur le sommeil, lourd et charnel ; la veille, dans cette perspective, se fait instrument d'ascèse et de pénitence, au même titre que les autres pratiques de mortification. Néanmoins il existe un sommeil d'un autre ordre, pénétré par la grâce, et précédé souvent de prières et de larmes ; ce sommeil réceptif, marqué par la séparation provisoire du corps et de l'âme, est le cadre de songes ou d'avisions. Paul Bretel précise alors les 
critères qui, dans les contes de la Vie des Pères, démarquent le songe de l'avision: alors que le songe advient nécessairement pendant le sommeil, l'avision peut se prolonger pendant l'état de veille; en outre, elle comporte un caractère plus concret que le songe, car elle est susceptible de laisser au dormeur une trace matérielle de son apparition. Ces deux formes de manifestations divines, qu'elles soient directement adressées au bénéficiaire ou qu'elles passent par un intermédiaire spécialiste du sacré, interviennent généralement lors d'une crise spirituelle, pour apporter le réconfort au pécheur et le remettre dans la droite voie.

Jean-René Valette, à partir de prémisses théoriques comparables, complète les catégorisations de Paul Bretel, tout en montrant comment elles confortent la cohérence de la Queste del Saint Graal. Alain de Lille distingue trois types de sommeil : le sommeil infra hominem, lié à la vita animalis, le sommeil secundum hominem, lié à la vita rationalis, et le sommeil supra hominem, lié à la vita spiritualis. Chaque type de sommeil reçoit une illustration dans la Queste. Le sommeil infra hominem, chargé de péchés charnels, est celui de Lancelot «entransés » au début de son parcours; c'est également celui que Perceval connaît sur l'île où le Diable, dans l'intention de le faire succomber à la tentation, se présente à lui sous la forme d'une belle demoiselle : prélude aux tentations du repas, du vin et de la luxure, il inaugure la chute (provisoire) du chevalier. Le sommeil secundum hominem, qui succède, dans l'ordre du récit, au sommeil infra hominem, est visité de songes, dont le symbolisme demeure accessible à la raison : il s'agit d'une forme encore voilée d'accès au divin, inscrite dans une corporéité et une histoire bien humaines. Les neuf songes de la Queste, situés dans sa partie médiane, illustrent donc la vita rationalis. La transition vers la vita spiritualis est marquée par l'apparition du cerf entouré des quatre évangélistes, à l'intersection de la vision sensible et de la révélation «aperte». Les trois cas de sommeil supra hominem, lieu des plus hautes extases, prennent place à la fin du roman : sommeil de Lancelot après l'arrêt à 
l'eau de Marcoise, dans la nef qui lui est envoyée ; " châtiment délicieux », pour reprendre les mots d'Albert Pauphilet, infligé au même Lancelot dans le château du Graal ; sommeil de Galaad, enfin, dans la Nef de Salomon. A Lancelot, par conséquent, il est donné de parcourir tous les degrés de spiritualité : on peut voir là le signe qu'il reste, plus que son fils, le héros véritable du cycle du LancelotGraal.

Ainsi, l'insomnie, le songe et le sommeil, phénomènes essentiellement physiologiques dans un certain nombre de textes, favorisent ailleurs le contact avec une transcendance qui peut prendre les couleurs de l'Autre Monde féerique ou bien de l'au-delà chrétien, permettant alors l'accès à une forme supérieure de spiritualité... à moins qu'ils ne jouxtent l'espace périlleux de l'oubli, de la désespérance et de la mort. Le rapport entre un régime nocturne, révélateur de vérité ou matrice de «resveries », et le régime diurne de la réalité, varie selon les textes et les contextes génériques - évolue également tout au long du Moyen Age. La diversité des exemples dont les communications ont tiré parti témoigne d'un va-et-vient constant entre plusieurs champs disciplinaires : textes médicaux (Jean-Marie Fritz), didactiques (Chantal Connochie-Bourgne), théologiques (Paul Bretel, Jean-René Valette), appel aux souvenirs du paganisme antique (Fabienne Pomel, Jean-Jacques Vincensini, Marylène Possamaï-Pérez) ou à un «folklore » lié aux fantasmes profonds d'une époque (Jelles Koopmans), ces croisements disent la richesse et la multiplicité des pistes qu'ont ouvertes ces rencontres rennaises. 